\title{
Grandes hidrelétricas na Amazônia: impactos no recurso pesqueiro e na pesca artesanal, desafios e lições aprendidas na bacia do Rio Madeira
}

\section{Large hydroelectric power plants in the Amazon: impacts on fisheries and fisheries management, lessons learned and challenges in the Madeira River basin}

\author{
Carolina Rodrigues da Costa Doria ${ }^{1}$ \\ Jynessa Dutka-Gianelli ${ }^{2}$ \\ Simone Athayde ${ }^{3}$ \\ Aidee Maria Moser Torquato Luiz ${ }^{4}$ \\ Walterlina Brasi 5 \\ Guillermo Moisés Bendezú Estupiñán ${ }^{6}$ \\ Marilia Hauser 7 \\ Paul André Van Damme 8 \\ Gislene Torrente-Vilara ${ }^{9}$
}

\begin{abstract}
Resumo: As consequências socioecológicas da implantação de grandes empreendimentos hidrelétricos são claras e transcendem fronteiras geopolíticas e disciplinares. Este trabalho sintetiza e compartilha relatos de profissionais de diversas áreas e populações atingidas por barragens hidrelétricas construídas na bacia do Rio Madeira. Os depoimentos, discussões e abordagens participativas, evidenciaram a necessidade de conexões e cooperação entre diversos atores, para trabalharmos na solução de problemas comuns gerados por grandes empreendimentos, com destaque para a gestão de recursos pesqueiros e pesca artesanal. A abrangência das percepções e experiências de vários atores podem contribuir para mitigação de impactos, por meio da convergência de conhecimentos e identificação de necessidades comuns.
\end{abstract}

Palavras-chave: pesquisa transdisciplinar, recursos pesqueiros, pesca artesanal, Rio Madeira, perspectiva dos atores, governança, águas internacionais,

\footnotetext{
${ }^{1}$ Universidade Federal de Rondônia, BR 364, km 9.5, Zona Rural, 76815-800, Porto Velho, RO, Brasil / Pós-graduação Desenvolvimento Regional e Meio Ambiente

${ }^{2}$ Michigan State University, Department of Community Sustainability, Manly Miles Building, room 205. 1405 S. Harrison Road, East Lansing, MI, 48823. USA.

${ }^{3}$ Amazon Dams Network, Tropical Conservation and Development Program, Center for Latin American Studies, University of Florida. 381 Grinter Hall, PO Box 115530, Gainesville, FL, US, 32611-5530.

${ }^{4}$ Ministério Público do Estado de Rondônia.

${ }^{5}$ Laboratório de Ictiologia e Pesca, Departamento de Biologia, Universidade Federal de Rondônia (UNIR), BR 364, km 9,5, 76801-059 Porto Velho, RO, Brasil.

${ }^{6}$ Wildlife Conservation Society - WCS Brasil. Rua Costa Azevedo 9, sala 403, Centro, 69010-230 Manaus, AM, Brasil.

${ }^{7}$ Universidade Federal de Rondônia, BR 364, km 9.5, Zona Rural, 76815-800, Porto Velho, RO, Brasil/ Grupo de Estudos e Pesquisas em Educação Superior/Observatório Educação Superior e Desenvolvimento.

${ }^{8}$ Faunagua, Av. Max Fernández, Sacaba - Cochabamba, Estado Plurinacional de Bolívia

${ }^{9}$ Universidade Federal de São Paulo, Departamento de Ciências do Mar, Campus Baixada Santista. Rua Doutor Carvalho de Mendonça, 144, Encruzilhada, 11070-100, Santos, SP, Brasil.
} 


\title{
pesca transnacional.
}

\begin{abstract}
The social-ecological consequences of the implementation of large hydroelectric projects are clear and transcend frontiers and disciplines. This work synthesizes and shares reports of professionals from diverse disciplinary areas and populations affected by hydroelectric dams built in the Madeira River basin. Through testimonials, discussions and participatory approaches, we highlight the need for connections and cooperation between different actors, to address common problems caused by large infrastructure projects, with an emphasis in fish resources and artisanal fisheries systems. The scope of the perceptions and experiences of diverse stakeholders can contribute to mitigate impacts, through the convergence of knowledges and the identification of common issues.
\end{abstract}

Keywords: transdisciplinary research, fish resources, artisanal fisheries, Madeira river, actors perspectives, governance, international waters, transnational fisheries.

\section{Introdução}

O aproveitamento do potencial de geração de energia hidráulica, viabilizado pela construção de usinas hidrelétricas, tem sido um caminho adotado como estratégia para a industrialização e o crescimento econômico de diversos países da América Latina (Soares-Filho et al. 2006). Na bacia Amazônica, 154 grandes hidrelétricas (>30 Megawatt, MW) estão em operação e 221 estão planejadas para as próximas décadas (Castello e Macedo 2015). No Brasil, o setor elétrico defende a implantação de hidrelétricas como uma saída à crescente demanda de eletricidade e destaca como pontos positivos do processo a segurança energética do país para seu desenvolvimento e a geração de empregos (MME/EPE 2017). Contudo, historicamente são inúmeros os relatos de impactos negativos de grandes hidrelétricas sobre os recursos naturais e sobre o modo de vida de comunidades afetadas pelo empreendimento e usuárias dos recursos naturais, representando um alto custo social, econômico e cultural para essas localidades (ANSAR et al. 2014, WINEMILLER et al. 2016). Especialmente em regiões tropicais e subtropicais, como na Amazônia, a qualidade de vida das comunidades locais depende estreitamente da conservação do recurso biológico (ALHO et al. 2012). A bacia do rio Madeira nos mostra um exemplo de grandes lições aprendidas sobre o estabelecimento de empreendimentos hidrelétricos na região tropical, através da análise de casos das barragens de Samuel, no rio Jamari (afluente do Madeira), e Jirau e Santo Antônio, no rio Madeira (Figura 1) com inúmeros impactos socioeconômicos e ambientais na região 
(FEARNSIDE 2014).

A implantação de grandes empreendimentos hidrelétricos tem como consequência a expressiva modificação de atributos hidrológicos, físico-químicos e biológicos do sistema fluvial, levando à alterações na composição e abundância de sua ictiofauna original e recursos pesqueiros (OLDEN e NAIMAN 2010, PETESSE e PETRERE 2012, ALHO et al. 2015, CASTELLO e MACEDO 2016, WINEMILLER et al. 2016). Na maioria dos casos, as alterações hidrológicas, biológicas e ecológicas resultam em impactos socioeconômicos relevantes (FEARNSIDE 1999, CEREGATO e PETRERE 2003, PETESSE e PETRERE 2012, ANDERSON et al. 2018), visto que a pesca constitui uma das principais fontes de proteína animal e de renda para as comunidades ribeirinhas amazônicas (CERDEIRA et al. 1997, ISAAC e ALMEIDA 2011; PEREZ et al. 2015).

O vasto conhecimento técnico-científico existente sobre impactos de hidrelétricas é de difícil extrapolação para a Amazônia. A alta diversidade exige profundo conhecimento na escala espaço-temporal adequada para cada empreendimento e para a mitigação dos impactos proporcionais a sua vasta dimensão geográfica, sendo que com frequência os impactos extrapolam os limites tradicionalmente estabelecidos nos documentos oficiais (incluindo questões transnacionais como na Bacia do Rio Madeira) e não consideram a percepção dos atores locais (DORIA et al. 2017). Esta realidade tem gerado inúmeros conflitos entre atores, resultando também em inúmeras ações contra as empresas construtoras, exigindo do poder judiciário e das agências que monitoram o licenciamento desses empreendimentos, um conhecimento interdisciplinar e transdisciplinar sobre diferentes temáticas.

A incorporação do conhecimento ecológico tradicional derivado das experiências de atores locais tem sido reconhecida como uma alternativa importante para coletar informações sobre problemas socioecológicos localizados, conflitos e conservação de populações de animais e seus habitats (Gilchrist et al. 2005, Raymond et al. 2010) mostrando o potencial do uso da informação obtida com e/ou pelos atores locais na avaliação de mudanças ambientais, deteção e mitigação de impactos, e gestão dos recursos (Huntington et al. 2004). Diferentes abordagens participativas visando ampliar o envolvimento dos atores locais no planejamento e na tomada de decisão em relação à questões am- 
bientais mostraram evidências de que uma ampla participação pode levar a melhores soluções para estes problemas (Reed 2008). Por exemplo, princípios de cogovernança pesqueira envolvendo parcerias entre órgãos governamentais e diversos parceiros estratégicos aplicados em comanejo de recursos pesqueiros e outros recursos (Ostrom 1990, Pomeroy e Berkes 1997, Fujita et al. 2010, Idhe et al. 2011, Haapasaari et al. 2012), e o envolvimento de atores locais na pesquisa, gestão participativa e processo de tomada de decisão, tem resultado na resolução de conflitos e melhoria do gerenciamento pesqueiro (Olsson et al. 2004).

Neste sentido, o presente estudo teve como objetivo analisar, por meio da perspectiva de diversos atores ou partes interessadas (stakeholders), os impactos socioambientais provocados pela instalação de empreendimentos hidrelétricos sobre os recursos pesqueiros, e as estratégias de gestão dos impactos sinérgicos dos empreendimentos de Santo Antônio e Jirau, em escala regional e transnacional. São elencados os desafios e lições apreendidas com as experiências vivenciadas pós-construção dos projetos, como forma de propor melhores práticas para as usinas já instaladas e futuras, integrando conhecimentos e experiências à realidade e necessidades locais.

\section{Metodologia}

\section{1. Área de estudo}

O rio Madeira é o maior tributário do rio Amazonas em vazão e transporte de sedimentos (Goulding 1979). Apresenta peculiaridades ambientais, destacando-se a imensa área de sua bacia, estimada em 1.380 .000 km2, distribuída em territórios do Brasil, Bolívia e Peru; abrigando ainda nesse trecho 19 corredeiras (CELLA-RIBEIRO et al. 2013) das quais a 3 foram afogadas pela construção de duas hidrelétricas em cascata: Jirau e Santo Antônio. Recentemente, este rio ficou conhecido por exibir a maior riqueza ictiíca do mundo inventariada em uma bacia hidrográfica, com mais de 1300 espécies de peixes descritas pela ciência (Ohara et al. 2010) o que representa quase metade das espécies válidas descritas para a Amazônia (TEDESCO et al. 2017:www.amazonfish.com). Ainda, o recurso pesqueiro oriundo da bacia do Madeira apresenta grande importância socioeconômica regional para os três países que a compartilham, gerando 
Tabela 1. Detalhes técnicos das usinas de Santo Antônio e Jirau na bacia do rio Madeira

\begin{tabular}{l|l|l}
\hline Caraterística & Jirau & Santo Antônio \\
\hline Tipo de usina: Hidroenergia & A fio d'agua -turbina a bulbo & A fio d'agua -turbina a bulbo \\
\hline Potência instalada (Megawatts) & 3750 & 3568 \\
\hline Área de superfície do reservatório (km2) & 362 & 422 \\
\hline Ano de fechamento do reservatório & 2012 & 2011
\end{tabular}

Fonte: http://www.santoantonioenergia.com.br/tecnologia/tecnologiaavancada/

proteína animal e renda para inúmeras comunidades ribeirinhas e pescadores comerciais locais (Doria et al. 2018a).

Atualmente, o sistema socioecológico da Bacia do rio Madeira encontra-se modificado, em função da operação dos aproveitamentos hidrelétricos de Jirau e Santo Antônio, respectivamente a terceira e quarta maior geradora hídrica do Brasil e dentre as 20 maiores hidrelétricas do mundo. O detalhe técnico de cada uma delas é apresentado na Tabela 1.

O escopo deste estudo abrange toda a bacia do Madeira na sua porção brasileira, boliviana e peruana (Figura 1).

\subsection{Fonte de dados e análise}

No presente estudo usamos como fontes de dados as apresentações orais e resumos do "Seminário e Oficina Internacional Brasil, Bolívia e Peru: Desafios Nacionais e internacionais de gestão dos recursos pesqueiros na bacia do Madeira", realizado pelo Ministério Público Estadual de Rondônia, em parceria com o Laboratório de Ictiologia e Pesca da Universidade Federal de Rondônia (LIP/UNIR) e com apoio da Rede Internacional de Pesquisa em Barragens Amazônicas /Amazon Dams Network (RBA/ADN), entre os dias 21 e 23 de maio de 2018 em Porto Velho (Doria et al. 2018b).

O referido seminário teve como objetivo geral promover o diálogo, por meio de uma abordagem transdisciplinar, entre representantes de diversas instituições e órgãos governamentais sobre a gestão dos recursos pesqueiros da bacia do rio Madeira frente aos impactos de empreendimentos hidrelétricos. O evento contou com participação de trinta e um (31) palestrantes representantes de diferentes grupos e regiões da bacia do Madeira (Brasil, Bolívia e Peru) e dos Estados Unidos, convidados para apresentar uma reflexão temática pautada em distintas áreas disciplinares sobre os impactos provocados 


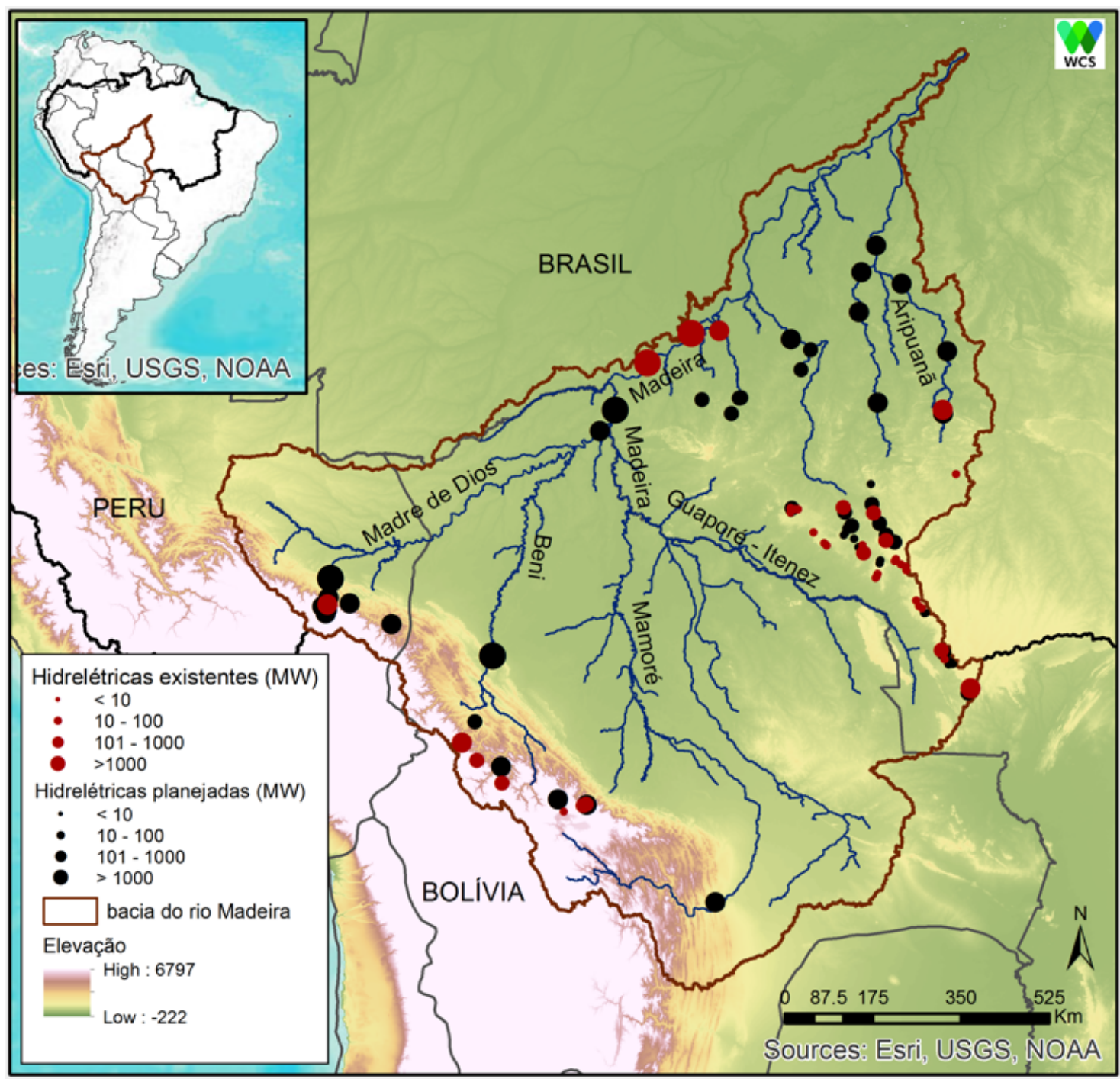

Figura 1. Bacia hidrográfica do rio Madeira, com indicação das usinas hidrelétricas existentes e em planejadas na região. Fonte: WCS Brasil

no recurso pesqueiro e na pesca nacional e transnacional como resultado da operação das UHEs do Madeira, incluindo também estudos de caso e experiências de outras bacias Amazônicas. Na sequência, os palestrantes participaram de uma oficina que teve como objetivo consolidar e compartilhar as experiências decorrentes das apresentações e debates ocorridos no seminário, bem como consolidar propostas de gestão da pesca para a bacia do rio Madeira (regional e transnacional).

Para o exercício e análises apresentados neste artigo, os palestrantes foram agrupados em cinco categorias: 1. usuários (pescadores), 2. técnicos ou gestores das agências de governo (federal e estadual), 3. técnicos ou gestores das agências não- 
Tabela 2. Grupos e subgrupos, país de origem, número de palestrantes e referências utilizadas para as palestras proferidas no "Seminário e Oficina Internacional -Brasil, Bolívia e Peru: Desafios nacionais e internacionais de gestão dos recursos pesqueiros na bacia do Madeira"

\begin{tabular}{|c|c|c|c|c|}
\hline $\begin{array}{l}\text { do IVadeir } \\
\text { Grupo }\end{array}$ & Sub-grupo & $\begin{array}{l}\text { País de ori- } \\
\text { gem }\end{array}$ & $\begin{array}{l}\text { Número de } \\
\text { palestran- } \\
\text { tes }\end{array}$ & Referência \\
\hline \multirow[t]{3}{*}{ Usuários } & Pescadores & Brasil & 3 & $\begin{array}{l}\text { Gomes }(2018) ; \quad \text { Silva } \\
\text { (2018); Moura (2018) }\end{array}$ \\
\hline & Pescadores & Bolívia & 1 & Rodrigues (2018) \\
\hline & Pescadores & Peru & 2 & Chuctaya (2018) \\
\hline \multirow{5}{*}{$\begin{array}{l}\text { Técnicos/Gestores } \\
\text { Públicos }\end{array}$} & Agência federal & Brasil & 2 & Estral (2018) \\
\hline & Agência estadual & Brasil & 1 & Lustosa (2018) \\
\hline & Agência estadual & Peru & 1 & Bardales (2018) \\
\hline & Agência federal & $\begin{array}{l}\text { Estados Uni- } \\
\text { dos }\end{array}$ & 2 & $\begin{array}{ll}\text { Melis (2018); } & \text { Yalucik } \\
(2018) & \end{array}$ \\
\hline & $\begin{array}{l}\text { Ministério publico } \\
\text { estadual }\end{array}$ & Brasil & 2 & Luiz (2018) \\
\hline \multirow[t]{4}{*}{ Sociedade civil } & $\begin{array}{l}\text { Colônia de Pesca- } \\
\text { dores }\end{array}$ & Brasil & 2 & $\begin{array}{lll}\begin{array}{l}\text { Veloso } \\
(2018)\end{array} & \text { (2018); } & \text { Melo } \\
\end{array}$ \\
\hline & $\begin{array}{l}\text { Organização não } \\
\text { governamental }\end{array}$ & Brasil & 3 & $\begin{array}{ll}\text { Leite (2018); } & \text { Souza } \\
(2018) & \end{array}$ \\
\hline & $\begin{array}{l}\text { Organização não } \\
\text { governamental }\end{array}$ & Bolívia & 1 & VanDamme (2018) \\
\hline & $\begin{array}{l}\text { Empresas constru- } \\
\text { toras }\end{array}$ & Brasil & 2 & $\begin{array}{l}\text { Candido(2018); Neto } \\
\text { (2018) }\end{array}$ \\
\hline \multirow[t]{4}{*}{ Academia } & Pesquisadores & Brasil & 5 & $\begin{array}{l}\text { Doria (2018); Torrente- } \\
\text { Vilara; (2018); Hau- } \\
\text { ser(2018); Cavalcante } \\
(2018)\end{array}$ \\
\hline & Pesquisadores & Peru & 1 & Pereya (2018) ; \\
\hline & Pesquisadores & $\begin{array}{l}\text { Estados Uni- } \\
\text { dos }\end{array}$ & 3 & $\begin{array}{l}\text { Kaplan (2018); } \text { Dutka- } \\
\text { Gianelli (2018); Athayde } \\
\text { (2018) }\end{array}$ \\
\hline & Total & 4 países & 31 & $\mathrm{~N} / \mathrm{A}$ \\
\hline
\end{tabular}

governamentais, 4. empresa construtora de empreendimentos hidrelétricos, 5. academia, de acordo com a nacionalidade e agência de origem (Tabela 2).

Considerando a literatura existente sobre impactos socioeconômicos e ambientais, governança e monitoramento dos recursos pesqueiros e da pesca frente à implantação de empreendimentos hidrelétricos, as palestras e resumos foram revisadas com o objetivo de identificar evidências de:

- impactos ambientais em escala local, regional e/ou transnacional (alterações hidrológicas, alterações na ocorrência e abundância de espécies; impactos sinérgi$\cos )$; 
- impactos sociais e econômicos; regionais e/ou transnacionais;

- lacunas ou deficiências e dificuldades no processo de gestão dos impactos provocados pelas obras na atividade pesqueira;

- lacunas ou deficiências e dificuldades no processo de monitoramento dos impactos.

As recomendações e/ou sugestões oferecidas pelos palestrantes ou grupos de trabalho da oficina foram consideradas como desafios para promover a sustentabilidade da pesca na bacia e foram agrupados seguindo o tema central ao qual estavam relacionadas:

- imitigação dos impactos nas comunidades pesqueiras afetadas e fortalecimento destas;

- legislação e gestão dos impactos;

- estudos de monitoramento dos impactos sobre o recurso pesqueiro e a pesca;

- devolutiva dos dados às populações afetadas;

- conservação do recurso pesqueiro nacional ou transnacional.

As evidências e/ou sugestões foram referenciadas com o sobrenome do palestrante (se a fonte for uma determinada palestra), ou com a sigla "OFC" (se a fonte for resultado da oficina). Para maiores detalhes ver o relatório e vídeos do evento disponíveis em: https://www.mpro.mp.br/web/caop-meio-ambiente/formulario.

\section{Resultados}

\subsection{Impactos ambientais}

Os impactos ambientais em escala local, regional e transnacional relatados foram:

(i) Alteração no fluxo e dinâmica do pulso hidrológico do rio como resultado do represamento do rio Madeira (TIMPE AND KAPLAN, 2017; Kaplan 2018). O autor ressalta que dentre os efeitos ecohidrológicos das barragens, as maiores alterações observadas estão relacionadas ao pulso de inundação, e que os efeitos cumulativos dessas alterações hidrológicas são amplificados em rios com múltiplas barragens, 
como no caso do rio Madeira. Os modelos matemáticos apresentados demonstraram que essas alterações podem afetar negativamente a produção pesqueira na bacia do Madeira em escala local e regional.

(ii) Alteração de acidentes geográficos naturais responsáveis por gerar parte da diversidade aquática, regular a movimentação das espécies e determinar a composição da ictiofauna. Torrente-Vilara (2018) ressalta a importância das cachoeiras do trecho de corredeiras, em especial Jirau e Teotônio, atuando como importante filtro ambiental para a ictiofauna local atualmente afogadas pela construção das UHEs do rio Madeira. Algumas espécies historicamente ultrapassam as cachoeiras; outras são limitadas por elas, mas, no geral, a composição das espécies de peixes é diferente acima e abaixo das cachoeiras. Lamentavelmente, as cachoeiras são o principal alvo de empreendimentos hidrelétricos que as afoga para formar grandes reservatórios, eliminando esse filtro ambiental que regula as espécies que devem ou não passar no sistema natural (Torrente-Vilara 2018). Assim, a construção de UHEs representauma modificação local nas condições ambientais (substituindo um ambiente correntoso com alta concentração de oxigênio por um ambiente lêntico ou semi-lêntico) afogando as cachoeiras e impedindo que elas sejam capazes de atuar como "filtro ambiental". A eliminação desse filtro interfere de forma distinta na conectividade entre os sistemas da jusante e montante. Por um lado, gera impactos relacionados a limitação da conectividade de espécies que utilizam toda a bacia para completar seu ciclo de vida, como é o caso da dourada (Brachyplatystoma rousseauxii). Ainda, haviam estoques genéticos de uma mesma espécie naturalmente limitados pelas cachoeiras de forma eficiente (exemplo, a sardinha Triportheus albus), o que promovia a biodiversidade dos estoques no sistema com fluxo gênico controlado pelas cachoeiras. Ainda, o sistema de transposição quando construído de forma diferente do modelo proposto (imitando o filtro ambiental) não atua com eficiência ecológica na migração de espécies que deveriam transpor os barramentos e pode facilitar invasão rio acima de espécies indesejáveis e historicamente restritas a parte baixa.

(iii) Alteração da composição e abundância de espécies-alvo da pesca comercial, especialmente de espécies migradoras, observados na porção brasileira (Doria 2018, 
Hauser 2018, Veloso 2018, Melo 2018, Gomes 2018, Moura 2018, Silva 2018), boliviana (Van Damme 2018, Rodrigues 2018) e peruana (Chuctaya 2018, Pereyra 2018). A despeito dos representantes da Usina de Jirau terem informado que até o momento não são observados impactos significativos na ictiofauna (Neto e Candido 2018), Hauser (2018) destacou o impacto da usina na migração dos bagres do gênero Brachyplatystoma, em particular a dourada (B. rousseauxii). A espécie realiza migrações em escala transnacional, desde a foz do rio Amazonas até a montante de seus tributários, incluindo o rio Madeira. Seu ciclo de vida envolve a reprodução nos Andes bolivianos com larvas e juvenis derivando até o estuário onde crescem e, posteriormente, sobem os afluentes do rio Amazonas quando adultos para se reproduzir, exibindo o comportamento de homing natal (retornam para se reproduzir na mesma região onde nasceram; Duponchelle et al. 2017). Hauser (2018) aplicou o método de análise biogeoquímica dos otólitos (estrutura óssea do ouvido dos peixes), utilizando a assinatura isotópica de estrôncio e selênio em otólitos, descrevendo evidências dos efeitos negativos das UHEs de Santo Antônio e Jirau na migração dessa espécie.

Além disso, a alteração na composição das espécies na área a montante do reservatório é agravada pelo aumento da abundância do pirarucu (Arapaima gigas), espécie carnívora invasora de grande porte, que foi introduzida acidentalmente por escapes de piscicultura na Amazônia boliviana e peruana nos anos 70 do século anterior (VAN DAMME et al. 2015), como apresentado nos estudos relatados de Catâneo et al. (2017) relatado por Doria (2018).

\subsection{Impactos sociais e econômicos}

(i) Diminuição da captura do pescado e de renda dos pescadores. As espécies migradoras são de grande importância na pesca comercial e para a subsistência das famílias ribeirinhas nos 3 países que compartilham a bacia do rio Madeira (Doria 2018, Rodrigues 2018, PereyRa 2018, Van Damme 2018, Chuctaya 2018). Contudo, os relatos descrevem a diminuição da captura do pescado e, consequentemente, da renda dos pescadores nas localidades à jusante, na área do reservatório, bem como à montante dos dois empreendimentos (relatos de pescadores presentes no evento, 
DORIA 2018).

Doria (2018) apresentou resultados dos estudos de Lima (2017) que demostram a diminuição na captura de algumas espécies na região de Porto Velho, com destaque para a dourada, que apresentou diminuição de $74 \%$ na média de captura anual quando comparou-se os anos pré e pós implantação das barragens. Estes resultados contradizem a informação apresentada pelos representantes da Usina de Jirau que, até o momento, o monitoramento conduzido pela empresa não registrou impactos significativos nas espécies migradoras (VerÍssimo e Candido 2018).

\subsection{Lacunas, deficiências e dificuldades no processo de gestão e mitigação dos impactos}

(i) Os documentos norteadores do processo de licenciamento ambiental, EIA/RIMA e os Planos Básicos Ambientais e Informação Técnica IT.060 do IBAMA (IBAMA 2008), relacionados à ictiofauna e à pesca, não atenderam de forma eficiente as necessidades dos pescadores (na porção brasileira da bacia são cerca de 1500 pescadores comerciais) e suas famílias, comprometendo a manutenção de suas atividades de geração de renda, cultura e costumes (Doria 2018, Cavalcante 2018, GOMES 2018, SILVA 2018, MOURA 2018). Os relatos apontam falhas no dimensionamento dos impactos, em especial nas localidades à jusante, além do não atendimento das condicionantes estabelecidas nos documentos (Doria 2018, Melo 2018, Veloso 2018, GOMES 2018, SILVA 2018, MOURA 2018; RAMOS et al. 2018).

(ii) A desestruturação das agências responsáveis pela gestão da pesca; a ausência de políticas públicas para o setor pesqueiro e de diálogo entre as instituições (governo e empreendedores) e atores dificultam ou impedem a resolução de problemas inerentes à atividade pesqueira antes e depois da implantação dos empreendimentos. Tal processo resultou no agravamento dos conflitos pesqueiros e na ausência de propostas factíveis e legítimas de mitigação do impacto dos empreendimentos (DORIA et al. 2017; Estral 2018, Nogueira 2018, Luiz 2018, Doria 2018, OFC)

(iii) Os pescadores brasileiros foram esquecidos ou ignorados no processo de licenciamento ambiental (DORIA et al. 2017). Os relatos demostram que os atores não foram ouvidos no momento de definir estratégias de mitigação dos impactos, bem como não tiveram seus pleitos atendidos. A exemplo, o deslocamento de pes- 
cadores para locais onde não tiveram condições de praticar a atividade pesqueira (MOURA 2018) ou restrição de área de pesca (GOMES 2018) levou à diminuição da renda obtida com a atividade e, em alguns casos, os pescadores foram levados a exercer atividade em locais de acesso restrito pela segurança das usinas (no "pé da barragem"). A pesca exercida ilegalmente em áreas proibidas como um último suspiro de manutenção da atividade pelos pescadores tem caracterizado a marginalização de pescadores que atuam historicamente (gerações) na pesca no rio Madeira (Doria 2018, Cavalcante 2018, GOMES 2018, SILVA 2018, MOURA 2018), o que é inaceitável do ponto de vista antropológico.

Os representantes das usinas de Jirau, por sua vez, relataram terem implementado um Grupo de Trabalho da Pesca na área afetada pela usina para discussão dos problemas relacionados à pesca, e informaram que há um projeto de manejo do pirarucu que está sendo desenvolvido como estratégia de mitigação dos impactos para uma das comunidades afetadas (Veríssimo e Candido 2018).

(iv) Apesar das críticas oficiais bolivianas à construção das barragens de Jirau e Santo Antônio, bem como do reconhecimento implícito nos estudos de linha de base de impactos futuros de barragens em peixes migratórios na Bolívia ou no Peru, esses impactos transfronteiriços não foram considerados no processo de licenciamento ambiental de ambas as barragens, mesmo tendo sido apontados como pontos frágeis do diagnóstico socioeconômico ambiental da ictiofauna e da pesca (FURNAS 2005).

\subsection{Lacunas, deficiências e dificuldades no processo de monitoramento dos impactos}

(i) Os Estudos de Impacto Ambiental (EIAs) bem como o Plano Básico Ambiental (PBA) das UHEs Santo Antônio e Jirau, no rio Madeira, não incluíram levantamentos de dados em territórios transnacionais. A limitação espacial de dados pretéritos aos impactos (tanto ecológicos quanto socioambientais na atividade pesqueira) bem como do monitoramento da construção, estabelecimento e operação das UHEs, impossibilita a delimitação adequada dos impactos e das medidas mitigadoras correspondentes (VAN DAMME 2018, DORIA 2018, TORRENTE-VILARA 2018). Os relatos de VAN DAMME (2018) e DORIA (2018) demonstram que essa ausência vai além 
das fronteiras brasileiras, visto que não foram realizados ou finalizados os estudos pré-implantação das usinas quanto aos impactos transnacionais sobre o recurso pesqueiro, e que estes impactos já estão sendo observados (VAN DAMME 2018, RODRIGUES 2018).

(ii) Disponibilização parcial ou ausente de dados primários obtidos nos programas de monitoramento (PBA) para uma análise em conjunto com a sociedade, que permita avaliar o impacto e a escala de abrangência (2003-2018), bem como uma discussão adequada e pautada em resultados concretos sobre o monitoramento pesqueiro, da ictiofauna e do sistema de transposição. Estes dados, quando existentes ou disponíveis, não são suficientes para avaliar os impactos dos empreendimentos. Além disso, a ausência de transparência sobre o monitoramento por parte das empresas tem originado relatos de fraudes de informação primária obtidas com os pescadores e outros atores relacionados à pesca (Moura 2018).

A exemplo, durante a reunião foi citada a ausência de discussão com a sociedade local, especialmente os afetados, em relação à eficiência dos sistemas de transposição, tanto da Usina Santo Antônio quanto da Usina de Jirau (Silva 2018, Melo 2018). A manutenção da migração de algumas espécies, que são importantes recursos pesqueiros, garante a manutenção das populações biológicas pois permite a espécie que complete o seu ciclo de vida. Ao ter a chance de completar seu ciclo de vida, as espécies migradoras que utilizam toda bacia do rio Madeira povoam naturalmente os rios da Bolívia e Peru (recurso transnacional) o que permitem a sustentabilidade da pesca nas áreas que historicamente utilizam tal recurso - à montante de Santo Antônio e Jirau. Portanto, disponibilizar dados sobre o monitoramento do sistema de transposição de ambos empreendimentos e aplicar técnicas que avaliem a efetividade da transposição de espécies que são importantes recursos transnacionais é uma ação fundamental para que se confirme (ou não) a efetividade do sistema de transposição, e que se discuta os resultados com a sociedade para eficiência na gestão da bacia (Hauser 2018, Rodrigues 2018, Van Damme 2018).

(iii) que a lei brasileira, boliviana e peruana preconize a gestão participativa da bacia do Madeira como unidade básica de gestão de seus recursos naturais. Nesse contexto, a gestão está pautada no uso comum dos recursos pesqueiros que, devido a alta 
mobilidade, requerem estratégias de gestão que contemplem toda área do ciclo de vida de algumas populações de peixes importantes para a pesca, o que muitas vezes ultrapassa os limites políticos de estados ou países, como no caso do Rio Madeira (Doria 2018).

\subsection{Desafios que ameaçam a sustentabilidade da pesca na bacia do Madeira}

A partir dos impactos relatados e das sugestões de gestão elencadas pelos palestrantes e participantes da oficina, foram identificados os desafios que ameaçam a sustentabilidade da pesca na bacia ameaçada por barragens:

\subsubsection{Quanto a mitigação dos impactos nas comunidades pesqueira afetadas e seu fortalecimento:}

1. Promover o cumprimento das condicionantes e programas de apoio à atividade pesqueira estabelecidos na licença de instalação (LI) e de operação (LO) dos empreendimentos, reconhecendo e redimensionando os impactos sobre a pesca e pescadores nas diferentes áreas da bacia, os quais extrapolam a área de impacto direto dos reservatórios (inclusive na área à jusante).

2. Promover o fortalecimento das colônias e organizações de apoio aos pescadores, e dos próprios pescadores para:

- garantir sua participação legítima nos processos de tomada de decisão para o ordenamento pesqueiro (DORIA et al. 2017; Doria 2018);

- prepará-los para o processo de negociação, consulta e gestão do recurso (OFC);

- articular o conhecimento local e o técnico-científico nos processos de tomada de decisão (Athayde 2018).

3. Promover o fortalecimento dos municípios e instituições locais para receber e administrar adequadamente os impactos locais.

4. Promover o fomento ao diálogo entre os atores, facilitando articulações institucionais e parcerias para fortalecer e capacitar comunidades locais, por meio de fóruns de pesca local, regional e internacional, possibilitando a integração dos saberes das comunidades tradicionais nos processos de planejamento das grandes 
obras e propondo a mitigação dos impactos na escala de bacia (Dutka-Gianelli 2018, Doria 2018).

5. Promover melhoria na cadeia produtiva, infraestrutura e condições higiênicosanitárias de pré-beneficiamento do produto; acesso a novos mercados que valorizem produtos obtidos de forma sustentável (por exemplo: um selo verde para pesca sustentável na Amazônia); e a capacitação de técnicos de instituições de extensão rural, com objetivo de transferir a responsabilidade técnica dos projetos de manejo (Souza 2018).

6. Promover o reconhecimento dos impactos transfronteiriços diretos e indiretos sobre os pescadores na Bolívia e Peru, incluindo a avaliação da viabilidade da implementação de mecanismos transnacionais de compensação ou mitigação.

\subsubsection{Quanto à legislação e gestão dos impactos:}

1. Criar um comitê de bacia regional e um transnacional para a bacia do Madeira (Brasil, Bolívia, Peru), a exemplo das usinas do rio Colorado (Melis 2018).

2. Estabelecer uma agenda comum entre os países, com ações que ajudem a articular a legislação pesqueira entre os países afetados por barragens, com foco na conservação das espécies migradoras; controle das espécies invasoras (Ex: 0 Pirarucu na Bolívia) e mitigação dos impactos das represas (Van Damme 2018).

3. Estabelecer estratégias de manejo adaptativo (ajustar/melhorar as práticas de gestão continuadamente de acordo com os resultados obtidos) na rotina das usinas implantadas e futuras, a partir do envolvimento ativo de diversos atores, considerando o status dos recursos avaliados e as necessidades locais, como estratégia para garantir a sustentabilidade da pesca nos 3 países afetados pelas usinas a exemplo das usinas de Lajeado na Bacia do Rio Tocantins (MARQUES 2018) e no rio Colorado (Melis 2018; Yalucky 2018). 


\subsubsection{Quanto aos estudos de monitoramento dos impactos sobre os recursos pes- queiros e a pesca:}

1. Fomentar a inclusão de estudos transnacionais (acordos diplomáticos de pesquisa colaborativa) para empreendimentos hidrelétricos bi ou trinacionais ainda durante a fase de inventário para avaliar o dimensionamento dos impactos na escala de bacia, especialmente com relação aos impactos no recursos pesqueiros e na pesca (OFC). Tal processo deve ser adotado através de um acordo com todos os países da Amazônia.

2. Garantir que o monitoramento dos recursos pesqueiros e da atividade pesqueira sejam contínuos, independentes e isentos (realizados por entidades do governo ou instituições de ensino pesquisa local com independência financeira do empreendimento), fornecendo informação por meio de cooperação técnica científica e governamental para investigar os impactos cumulativos e sinérgicos; consolidando e apoiando a pesquisa científica de qualidade e formação de uma comunidade científica em condições de atuação permanente neste monitoramento e na consolidação de uma base de dados compartilhada (DORIA et al. 2017).

3. A avaliação dos impactos deve considerar o conjunto de dados temporais que inclua toda fase pré e pós-UHEs e sinérgicos dos empreendimentos em toda a bacia do Madeira (UHE Santo Antônio; Jirau; Tabajara, Samuel e futuras) e identificar alternativas aos impactos sobre peixes, pesca e pescadores (Doria 2018, Torrente-Vilara 2018).

\subsubsection{Quanto à devolutiva dos dados às populações afetadas:}

- Implantar um comitê de acompanhamento da coleta de dados do monitoramento e compartilhamento de dados obrigatório, em linguagem acessível e transparente, para as comunidades atingidas pelos impactos e para a ciência em diferentes temáticas envolvidas (Lima 2018, Doria 2018, Torrente-Vilara 2018).

- Fomentar a apropriação das informações pelos atores por meio de abordagens metodológicas participativas como a ciência cidadã e fóruns de pesca locais, regionais e internacionais (Lima 2018), dentre outros formatos que possam ser desen- 
volvidos, discutidos e articulados em um processo de interação horizontal entre estes atores.

- Promover a formação de canais de transferência de informação de base e de monitoramento de dados entre os atores diplomáticos, técnicos e locais nos diferentes países impactados.

\subsubsection{Quanto à conservação do recurso pesqueiro nacional ou transnacional}

1. Manter e conservar o último fragmento de filtro ambiental representado no trecho de rio entre as cachoeiras de Jirau e Cachoeira Esperanza (Torrente-Vilara 2018). O fragmento relictual com 12 corredeiras daquele trecho do rio tem sido apontado como o principal fator ambiental que possivelmente está mantendo a oxigenação da calha do rio para as espécies migradoras e reofílicas (afinidade por ambientes com alta concentração de oxigênio) e, possivelmente, contribuindo também para regular a invasão de espécies a montante (aquelas que naturalmente só ocorrem na parte baixa do rio Madeira e, portanto, representarão invasão biológica caso atinjam a parte alta da bacia). Assim, a manutenção desse trecho de corredeiras é de importância crítica para garantir a manutenção de parte dos processos ecológicos fundamentais para conservação da ictiofauna bacia do rio Madeira, devendo ser protegida.

2. Garantir o funcionamento efetivo dos sistemas de transposição de peixes das UHEs de Santo Antônio e Jirau, para que os homers $\$^{10}$ e strayers $\$^{11}$ da dourada continuem tendo acesso as áreas reprodutivas de cabeceira e, assim, garantir a manutenção de populações viáveis nessa área, bem como sua contribuição aos estoques genéticos da bacia amazônica como um todo (Hauser 2018).

3. Implementar planos de conservação e manejo integrados que englobem toda área de ciclo de vida da dourada e de outros peixes migradores, independente dos limites geopolíticos, de país ou mesmo de estado.

4. Incentivar a pesca manejada do pirarucu invasor na área do reservatório como forma de diminuir a competição intra e interespecífica.

\footnotetext{
${ }^{10}$ https://www.mpro.mp.br/web/caop-meio-ambiente/formulario

${ }^{11}$ Strayers: peixes que falham ao reconhecer os rios em quem nasceram no momento da reprodução
} 


\section{Discussão}

Os testemunhos compartilhados durante o evento evidenciaram a ocorrência de impactos socioeconômicos significativos no recurso pesqueiro e na pesca artesanal na bacia do Madeira, tanto em território brasileiro quanto transnacional, em concordância com o que vem sido descrito na literatura (Winemiller et al. 2016). Dentre os impactos mais facilmente previstos e assumidos ao licenciar uma hidrelétrica, estão a alteração na composição e abundância das espécies de peixes nas áreas de influência direta e indireta dos empreendimentos, bem como a consequência dessas alterações para as comunidades ribeirinhas e pescadores. Tal efeito foi previsto no Diagnóstico Socioeconômico Ambiental dos Empreendimentos Hidrelétricos propostos para o rio Madeira, em 2005, juntamente com um conjunto de ações que deveriam ser adotadas para mitigar os impactos previstos (Vide: Componente Ictiofauna, Resumo Executivo em FURNAS 2005). As ações apresentadas foram reforçadas na Informação Técnica do órgão licenciador (I.T. No. 060/2008 - COHID/CGENE/DILIC/IBAMA) que apresenta diretrizes ao Programa de Monitoramento da Atividade Pesqueira da Usina Hidrelétrica de Santo Antônio, concluindo que:

Tendo em vista a especificidade da atividade pesqueira e a sua importância no contexto da economia na região do Madeira e seus afluentes e a necessidade de acompanhar a questão da pesca do ponto de vista social, incluindo considerações sobre possíveis modificações na estrutura, riqueza e abundância fauna aquática, deve ser apresentado um Programa de Compensação Social da Atividade Pesqueira com o objetivo de acompanhar a situação da atividade pesqueira, tendo como foco: (i) a manutenção da atividade pesqueira, cujo monitoramento é ação contínua; (ii) a compensação social da atividade pesqueira impactada, tendo como base os resultados do monitoramento contínuo, mediante a implantação de projetos que garantam a sustentabilidade do trabalho e da renda dos pescadores, permitindo a permanência na atividade ou a inserção, somente quando interessar, e desde que comprovada a viabilidade econômica, em atividades alternativas; (iii) a definição de um novo padrão tecnológico, incluindo ações para reestruturação da atividade, sempre que necessário.

O fomento de projetos locais voltados ao fortalecimento da atividade de pesca artesanal e ao uso sustentável dos recursos pesqueiros deve ser empreendido mediante ações de educação ambiental, utilizando metodologias participativas, com vistas a garantir que os projetos selecionados sejam demandas reais das comunidades e estejam ligados aos principais problemas relacionados à pesca. A proposição deve ser tanto a de compartilhamento de poder como de co-responsabilidade na implementação e posterior manutenção dos projetos. 
O não cumprimento ou cumprimento parcial dessas diretrizes é declarado nas falas dos atores presentes no evento e também no volume de ações judiciais protocoladas pelos atores e seus representantes junto aos órgãos competentes (Ação Civil Pública 0008477-07.2016.4.01.4100; Inquérito Policial 0135/2015SR/DPF/RO; Petição criminal n 1001770-35.2018.4.01.4100; Inquérito Civil 1.31.000.000214/2011-15; Laudo Pericial 1165/2016/CRP4/SEAP/MPF). O fato demostra que as normas e políticas norteadoras do processo de licenciamento existentes são frequentemente ignoradas e desrespeitadas e que os pescadores são invisibilizados no processo de implantação de grandes hidrelétricas na Amazônia (DORIA et al., 2017). Os pescadores, assim como outras comunidades tradicionais, são deixados de fora do processo de tomada de decisão na busca de alternativas para o novo cenário imposto a partir da inserção dos empreendimentos em seu território (REZENDE 2009, JAICHAND e SAMPAIO 2013, Athayde 2014).

Os pescadores, principais interessados, são paradoxalmente os mais vulneráveis dentro do debate. Os resultados dos impactos e os estudos produzidos tanto pela comunidade científica, ou mesmo a partir do conhecimento das comunidades, demostram que os atores estão isolados e esquecidos no atual modelo de governança. Com a barragem, viver da pesca ou viver como pescador é uma crise pessoal (e cultural), portanto, não se trata exclusivamente de um prejuízo econômico mas, também, de subsistência e identitário.

A pesca é uma atividade que não está somente refletida como um meio de subsistência, mas é um modo de vida (BRASIL et al. 2011). Nesse sentido, alterar a condição da pesca pela modificação antropogênica do ambiente utilizado na obtenção do recurso (rio) ou deslocar comunidades, altera locais de pesca historicamente mapeados ao longo de gerações de pescadores. Limitar o acesso dos pescadores ao recurso pesqueiro é o mesmo que impor um novo modo de vida. A atividade da pesca é uma tradição familiar que embute valores em relação ao uso e respeito do ambiente, ao peixe, como atividade que não tem relação com o compromisso de produzir em larga escala. A visão empreendedora que acumula divisas a partir da produção é secundária do ponto de vista do indivíduo e comumente sem nenhum valor para ele, o que gera conflito para políticas desse setor. A visão produtivista propagada por alguns atores e políticas governamentais não será adotada pelo pescador que tem na sua atividade uma arte passada de geração 
para geração. A visão distinta representada por um abismo entre a sociedade civil e sua forma de vida comparada aos atores da atividade pesqueira sobre o que representa o recurso natural tem sido tristemente desgastada, em grande parte, em função da negação (por parte de representantes do governo e da sociedade civil) sobre o potencial produtivo de cunho altamente ecológico possível de ser protegido e valorizado pela manutenção da atividade pesqueira.

\section{Conclusão}

Os testemunhos durante o evento proporcionaram evidências quanto às diferentes perspectivas representadas entre os pescadores, empresas, governo, academia e sociedade civil.

Muitos impactos são exacerbados por problemas já observados nos relatos e coadunam com os observados em outras regiões, tais como:

- ausência de informações adequadas para apoiar o planejamento, delimitação e mitigação do impacto bem como para a tomada de decisões (Kirchherr et al. 2016, Doria et al. 2017);

- deficiências no processo de participação e retorno das informações às partes interessadas (Athayde et al. 2016, Doria et al. 2017);

- ausência de estrutura governamental para gerenciar e apoiar os pescadores antes e após a construção de barragens (Doria et al. 2017).

Uma das grandes dificuldades se aloja na falta de oportunidades de comunicação e acompanhamento do processo para os atingidos e interessados, tanto nacional como transnacionalmente. O incentivo para a participação dos atores essenciais em cada fase específica no processo de planejamento e licenciamento, bem como a integração dos saberes destes - considerando as necessidades e características locais - são ferramentas imprescindíveis para a criação de um sistema inovador que possibilite a participação efetiva dos interessados.

O envolvimento de diversos atores sociais, representando órgãos federais, estaduais, regionais e municipais, em conjunto com empreendedores, acadêmicos e população local atingida pelos empreendimentos hidrelétricos, é um componente importante a 
ser observado nos processos de mitigação e principalmente, em discussões de planejamento para implementação de novas usinas. Uma abordagem envolvendo a participação social e favorecendo a possibilidade de diálogo e a cooperação entre os atores (tanto regionalmente como transnacionalmente) pode contribuir para a definição de ações apropriadas para os problemas locais, melhoria na qualidade de vida da população atingida, diminuição dos impactos negativos causados, e também, para a diminuição de conflitos entre os atores (Lima et al. 2015; Araujo e Moret 2016).

Os conflitos apontados durante o evento - de interesse, de atendimento, e de visibilidade institucional - expressam que a base está nas lacunas ou inconsistências no sistema de governança e tomada de decisão ao longo de todo o processo de licenciamento e monitoramento do empreendimento, e ressaltam o quanto há de ser percorrido.

Neste cenário, o Estado tem um importante papel na busca de soluções para os conflitos e deve investir esforços para:

- Promover o diálogo entre as partes interessadas, nacionalmente e trasnacionalmente, incluindo os pescadores, gestores e construtores, para que os recursos sejam administrados em escala de bacia;

- Executar, práticas de monitoramento dos projetos e programas de compensação por meio de instituições de pesquisa ou agências ambientais;

- Averiguar, com apoio da comunidade científica, as denúncias de adulteração de dados feitas pelos pescadores e seus representantes durante o evento;

- Fomentar práticas de gestão adaptativa e participativa que garantam a sustentabilidade do recurso e consequentemente da atividade da pesca e das comunidades que deles dependem.

Os pescadores e seus representantes, por sua vez, devem se fortalecer para cobrar sua participação na tomada de decisão e processos de gestão, bem como maior clareza nos processos de planejamento e licenciamento.

\section{Agradecimentos e Apoio}

Os autores agradecem ao Ministério Publico do Estado de Rondônia, a CAPES: Projeto Pró-Amazônia e Auxílio n. 3326/2013 e ao National Science Foundation (NSF): 
Apoio $n^{0}$ 1617413. Quaisquer opiniões, descobertas, conclusões ou recomendações contidas neste material são de responsabilidade exclusiva dos autores, e não necessariamente refletem as opiniões da National Science Foundation.

\section{Referências}

ALHO, C. J. R. Importance of biodiversity for the human health: an ecological perspective. Estudos Avançados, n.26, p. 151-165, 2012

Anderson, E. P. et al. Fragmentation of Andes-to-Amazon connectivity by hydropower dams. Science Advances, v.4, n.1, p.1-8. 2018.

ANSAR, A. et al. Should we build more large dams? The actual costs of hydropower megaproject development. Energy Policy, v. 69, p. 43-56, jun. 2014.

ARAUJO, N. C.; MORET, A. D. S. Direitos Humanos e Hidrelétricas: uma análise dos impactos socioambientais e econômicos gerados em Rondônia. Veredas Do Direito: Direito Ambiental e Desenvolvimento Sustentável, v. 13, n. 26, p. 167, 2016.

Athayde, S. Abordagens Participativas para o monitoramento da pesca com comunidades indígenas afetadas por Belo Monte. In: Doria, C. R. C.; Athayde, S.;

Dutka-Gianelli, J.; Luiz, A.M.T. Relatório: Seminário e Oficina Internacional Brasil, Bolívia e Peru: Desafios Nacionais e Internacionais de Gestão dos Recursos Pesqueiros na Bacia do Madeira. Ministério Público Estadual de Rondônia: Porto Velho, Maio de 2018.

ATHAYDE, S. et al. Collaborative learning, transdisciplinarity and social-environmental management in the Amazon: approaches to knowledge production between academia and society. Revista Brasileira de Pós-Graduação, v. 10, n. 21, 2014.

Athayde, S., Moreira P. F.; Heckenberger, M. Public feedback at risk in Brazil. Science, n.353, p.1217-1217. 2016

Bardales, J.F. L. La pesqueira em Madre Dios. Dirección Regional de la Producción DIREPRO Madre de Dios (Peru). In: Doria, C. R. C.; Athayde, S.; Dutka-Gianelli, J.; Luiz, A.M.T. Relatório: Seminário e Oficina Internacional Brasil, Bolívia e Peru: Desafios Nacionais e Internacionais de Gestão dos Recursos Pesqueiros na Bacia do Madeira. Ministério Público Estadual de Rondônia: Porto Velho, Maio de 2018.

BRASIL, W. SIMÃO, B.; SOBREIRO, T. Pesca. Pescadores. Pescaria. Meios e modos de vida na Amazônia. (Relatório de Trabalho. Projeto de Aplicação Ribeirinhos). Porto Velho, RO, 2011. Manuscrito.

Calvalcante, M. Hidrelétricas e comunidades ribeirinhas no alto rio Madeira- RO: uma análise sobre capacidade de resiliências das comunidades reassentadas. - GEOPLAM/UNIR Faunágua (Bolívia). In: Doria, C. R. C.; Athayde, S.; Dutka-Gianelli, J.; Luiz, A.M.T. Relatório: Seminário e Oficina Internacional Brasil, Bolívia e Peru: Desafios Nacionais e Internacionais de Gestão dos Recursos Pesqueiros na Bacia do Madeira. Ministério Público Estadual de Rondônia: Porto Velho, Maio de 2018.

CAPELARI, M. G. M.; CALMON, P. C. D. P.; ARAÚJO, S. M. V. G. Vincent e Elinor Ostrom: 
duas confluentes trajetórias para a governanca de recursos de propriedade comum. Rev. Ambiente \& Sociedade, São Paulo, v. XX, n. 1, p. 207-226, jan.-mar. 2017

CASTELLO, L.; MACEDO, M. N. Large-scale degradation of Amazonian freshwater ecosystems. Global Change Biology, v. 22, n. 3, p. 990-1007, mar. 2016.

CATÂNEO, D. T. B. S Invasão de Pirarucu (Arapaima gigas) em localidades à montante da cachoeira do Teotônio. In: I Simpósio de Desenvolvimento Regional e Meio Ambeite. Anais. UNIR:Porto Velho, 2017.

Cella-Ribeiro, A., Torrente-Vilara, G., Hungria, D. B.; Oliveira, M. (2013). As corredeiras do rio Madeira. In Peixes do rio Madeira (Queiroz,J.L. et al, eds). São Paulo: Editora Dialeto 2013.

CERDEIRA, R. G. P.; RUFFINO, M. L.; ISAAC, V. J. Consumo de pescado e outros alimentos pela população ribeirinha do lago Grande de Monte Alegre, PA - Brasil. Acta Amazonica, v.27, n.3, p. 213-228. 1997.

CEREGATO, S.A.; PETRERE Jr., M. Financial comparisons of the artisanal fisheries in Urubupungá complex in the middle Paraná river (Brazil). Brazilian Journal of Ecology, v.63, n.4, p.673-682, 2003.

Chuctaya, W. Representante dos pescadores de Puerto Maldonado (Peru). In: Doria, C. R. C.; Athayde, S.; Dutka-Gianelli, J.; Luiz, A.M.T. Relatório: Seminário e Oficina Internacional Brasil, Bolívia e Peru: Desafios Nacionais e Internacionais de Gestão dos Recursos Pesqueiros na Bacia do Madeira. Ministério Público Estadual de Rondônia: Porto Velho, Maio de 2018.

DORIA, C. R. C. et al. The invisibility of fisheries in the process of hydropower development across the Amazon. Ambio, 5 dez. 2017.

DORIA, C. R. C. 2018 A influência das hidrelétricas no sistema da pesca na bacia do rio Madeira Laboratório de Ictiologia e Pesca (UNIR - Brasil)/ ECOPORÉ. In: Doria, C. R. C.; Athayde, S.; Dutka-Gianelli, J.; Luiz, A.M.T. Relatório: Seminário e Oficina Internacional Brasil, Bolívia e Peru: Desafios Nacionais e Internacionais de Gestão dos Recursos Pesqueiros na Bacia do Madeira. Ministério Público Estadual de Rondônia: Porto Velho, Maio de 2018.

DORIA, C. R. C. et al. Review of Fisheries Resource Use and Status in the Madeira River Basin (Brazil, Bolivia, and Peru) Before Hydroelectric Dam Completion. Reviews in Fisheries Science \& Aquaculture, v. 26, n. 4, p. 494-514, 2 out. 2018 a.

Doria, C. R. C.; Athayde, S.; Dutka-Gianelli, J.; Luiz, A.M.T. Seminário e Oficina Internacional Brasil, Bolívia e Peru: Desafios Nacionais e Internacionais de Gestão dos Recursos Pesqueiros na Bacia do Madeira. Relatório. Ministério Público Estadual de Rondônia: Porto Velho, 2018b.

DUPONCHELLE, F. et al. Trans-Amazonian natal homing in giant catfish. Journal of Applied Ecology, v. 53, n. 5, p. 1511-1520, out. 2016.

Dutka-Gianelli, J. A. R. Experiências dos Fóruns de Pesca na Flórida. In: Doria, C. R. C.; Athayde, S.; Dutka-Gianelli, J.; Luiz, A.M.T. Relatório: Seminário e Oficina Internacional Brasil, Bolívia e Peru: Desafios Nacionais e Internacionais de Gestão dos Recursos 
Pesqueiros na Bacia do Madeira. Ministério Público Estadual de Rondônia: Porto Velho, Maio de 2018.

Estral, A. Realidade da Pesca Frente a Instalação das usinas Hidrelétricas em Rondônia. Um relato do Escritório da Secretaria Especial de Aquicultura e Pesca - Presidência da República. SEAP/RO. In: Doria, C. R. C.; Athayde, S.; Dutka-Gianelli, J.; Luiz, A.M.T. Relatório: Seminário e Oficina Internacional Brasil, Bolívia e Peru: Desafios Nacionais e Internacionais de Gestão dos Recursos Pesqueiros na Bacia do Madeira. Ministério Público Estadual de Rondônia: Porto Velho, Maio de 2018.

FEARNSIDE, P.M. Impacts of Brazil's Madeira River Dams: Unlearned lessons for hydroelectric development in Amazonia. Environmental Science and Policy, n.38, p.164-172. 2014

FEARNSIDE, P.M. Social impacts of Brazil's Tucuruí Dam. Environmental Management 24: 483-495. 1999.

Fujita, R. M., Honey, K. T., Morris, A., Wilson, J. R., \& Russell, H. Cooperative strategies in fisheries management: integrating across scales. Bulletin of Marine Science, v.86, n.2, p. 251-271. 2010.

FURNAS (Furnas Centrais Elétricas, S.A.), CNO (Construtora Noberto Odebrecht, S.A.), \& Leme, Engenharia (2005). EIA - Estudo de Impacto Ambiental Aproveitamentos Hidrelétricos Santo Antoônio e Jirau, Rio Madeira-RO. 6315-RT-G90-001. FURNAS, CNO, Leme Engenharia, Rio de Janeiro, RJ, Brazil. 8 Vols. Irregular Pagination.

Gilchrist, G., Mallory, M., \& Merkel, F. Can local ecological knowledge contribute to wildlife management: Case studies of migratory birds. Ecology and Society, v.10, n.1, p. 20. 2005.

GOMES, Z. R. Reflexão dos pescadores da região do São Sebastião. In: Doria, C. R. C.; Athayde, S.; Dutka-Gianelli, J.; Luiz, A.M.T. Relatório: Seminário e Oficina Internacional Brasil, Bolívia e Peru: Desafios Nacionais e Internacionais de Gestão dos Recursos Pesqueiros na Bacia do Madeira. Ministério Público Estadual de Rondônia: Porto Velho, Maio de 2018.

GOULDING, M. Ecologia da pesca do rio Madeira. Manaus: INPA, 172 p. 1979 Haapasaari, P., Mäntyniemi, S., Kuikka, S. Baltic herring fisheries management: Stakeholder views to frame the problem. Ecology and Society, v.17., n.3, 2012.

HAUSER, M. Migração da Dourada: a importância da conectividade dos rios Amazônicos. In: Doria, C. R. C.; Athayde, S.; Dutka-Gianelli, J.; Luiz, A.M.T. Relatório: Seminário e Oficina Internacional Brasil, Bolívia e Peru: Desafios Nacionais e Internacionais de Gestão dos Recursos Pesqueiros na Bacia do Madeira. Ministério Público Estadual de Rondônia: Porto Velho, Maio de 2018.

Huntington, H., Callaghan, T., Fox, S., Krupnik, I. Matching traditional and scientific observations to detect environmental change: a discussion on Arctic terrestrial ecosystems. Ambio, Special Report n.13 p.18-23, 2004.

IBAMA Informação Técnica I.T. No.060/2008 (COHID/CGENE/DILIC/IBAMA), IBAMA, Brasília, 2008. 
Ihde, T. F., Wilberg, M. J., Loewensteiner, D. A., Secor, D. H., \& Miller, T. J. The increasing importance of marine recreational fishing in the US: challenges for management. Fisheries Research, v.108, n.2, p.268-276, 2011.

Isaac V.J., de Almeida M.C. El consumo de pescado en la Amazonia brasileña. FAO, COPESCAAL/OP13: Roma. 54 pp. 2011.

Jaichand, V.; Sampaio, A.A. Dam and be damned: The adverse impacts of belo monte on indigenous peoples in Brazil. Human Rights Quarterly. n.35, p.408-447, 2013.

Kaplan, D. Os Efeitos das Barragens na Hidrologia dos Rios Amazônicos. In: Doria, C. R. C.; Athayde, S.; Dutka-Gianelli, J.; Luiz, A.M.T. Relatório: Seminário e Oficina Internacional Brasil, Bolívia e Peru: Desafios Nacionais e Internacionais de Gestão dos Recursos Pesqueiros na Bacia do Madeira. Ministério Público Estadual de Rondônia: Porto Velho, Maio de 2018.

KIRCHHERR, J.; POHLNER, H.; CHARLES, K. J. Cleaning up the big muddy: A metasynthesis of the research on the social impact of dams. Environmental Impact Assessment Review, v. 60, p. 115-125. 2016.

LEITE, G. Projeto de Ciência Cidadã para Amazônia (WCS). In: Doria, C. R. C.; Athayde, S.; Dutka-Gianelli, J.; Luiz, A.M.T. Relatório: Seminário e Oficina Internacional Brasil, Bolívia e Peru: Desafios Nacionais e Internacionais de Gestão dos Recursos Pesqueiros na Bacia do Madeira. Ministério Público Estadual de Rondônia: Porto Velho, Maio de 2018.

Lima, A.M.T., E.E. Marques, M.H. Ertzogue, D.T.A.M. Ferreira, and J.D. Lima. Os Rios Amazônicos Convertidos Em Gigawatts: Participação Social No Processo de Implantação de Usinas Hidrelétricas. Revista de Administração e Negócios Da Amazônia 7 (2): 136-58, 2015.

LIMA, M. A. L. História do ecossistema e dos recursos pesqueiros frente a implementação de hidrelétricas na bacia do rio Madeira. Doutorado - Porto Velho: Universidade Federal de Rondônia, 2017.

LUIZ, A.M.M.T 2018. Visão do Ministério Publico do Estado de Rondônia sobre Impactos Ambientais das Usinas do Madeira. In: Doria, C. R. C.; Athayde, S.; Dutka-Gianelli, J.; Luiz, A.M.T. Relatório: Seminário e Oficina Internacional Brasil, Bolívia e Peru: Desafios Nacionais e Internacionais de Gestão dos Recursos Pesqueiros na Bacia do Madeira. Ministério Público Estadual de Rondônia: Porto Velho, Maio de 2018.

Marques, E. E. Programa de monitoramento da ictiofauna na UHE Lajeado, Rio Tocantins. In: Doria, C. R. C.; Athayde, S.; Dutka-Gianelli, J.; Luiz, A.M.T. Relatório: Seminário e Oficina Internacional Brasil, Bolívia e Peru: Desafios Nacionais e Internacionais de Gestão dos Recursos Pesqueiros na Bacia do Madeira. Ministério Público Estadual de Rondônia: Porto Velho, Maio de 2018.

MELIS, T. S. Origem do Programa de Gestão Adaptativa da Barragem de Glen Canyon em Resposta ao Movimento Ambiental nos Estados Unidos: Um estudo de caso sobre o valor das enchentes de 1983 no Grand Canyon como um evento de foco para melhorar as operações de barragens (USGS - USA) In: Doria, C. R. C.; Athayde, S.; Dutka-Gianelli, J.; Luiz, A.M.T. Relatório: Seminário e Oficina Internacional Brasil, Bolívia e Peru: Desafios 
Nacionais e Internacionais de Gestão dos Recursos Pesqueiros na Bacia do Madeira. Ministério Público Estadual de Rondônia: Porto Velho, Maio de 2018.

MELO, G. Presidente da Colônia de Pescadores de Guajará Mirim (Brasil). In: Doria, C. R. C.; Athayde, S.; Dutka-Gianelli, J.; Luiz, A.M.T. Relatório: Seminário e Oficina Internacional Brasil, Bolívia e Peru: Desafios Nacionais e Internacionais de Gestão dos Recursos Pesqueiros na Bacia do Madeira. Ministério Público Estadual de Rondônia: Porto Velho, Maio de 2018.

MOURA, V. S. - Reflexão dos pescadores do Abunã. In: Doria, C. R. C.; Athayde, S.; Dutka-Gianelli, J.; Luiz, A.M.T. Relatório: Seminário e Oficina Internacional Brasil, Bolívia e Peru: Desafios Nacionais e Internacionais de Gestão dos Recursos Pesqueiros na Bacia do Madeira. Ministério Público Estadual de Rondônia: Porto Velho, Maio de 2018.

MME/EPE, 2017. Plano Decenal de Expansão de Energia 2026 / Ministério de Minas e Energia. Empresa de Pesquisa Energética. Brasília: MME/EPE, 2017.

Neto, V. e Candido, M. 2108. Programas Ambientais envolvendo a Ictiofauna na área de influência da UHE Jirau. In: Doria, C. R. C.; Athayde, S.; Dutka-Gianelli, J.; Luiz, A.M.T. Relatório: Seminário e Oficina Internacional Brasil, Bolívia e Peru: Desafios Nacionais e Internacionais de Gestão dos Recursos Pesqueiros na Bacia do Madeira. Ministério Público Estadual de Rondônia: Porto Velho, Maio de 2018.

OHARA, W. M. et al. Fish collection of the Universidade Federal de Rondônia: its importance to the knowledge of Amazonian fish diversity. Acta Scientiarum. Biological Sciences, v. 37, n. 2, p. 251-258, 2015.

Olden, J. D., Naiman, R. J. Incorporating thermal regimes into environmental flows assessments: modifying dam operations to restore freshwater ecosystem integrity. Freshwater Biology, n.55, p. 86-107, 2010.

Olsson, P., Folke, C., \& Berkes, F. Adaptive co-management for building resilience in social-ecological system. Environmental management, v. 34, n.1., p. 75-90, 2004.

Ostrom, E. A diagnostic approach for going beyond panaceas. Proceedings of the National Academy of Sciences, v.104, n.39, p.15181-15187, 2007.

Ostrom, E. Governing the Commons: The Evolution of Institutions for Collective Action. Cambridge: Cambridge University Press, 1990.

PÉREZ T., ZAMBRANA V., VAN DAMME P.A., CAROLSFELD J. (2014). Consumo de pescado en la Amazonia boliviana. pp. 289-338. En: MRE-MMAyA (Eds.). Sistemas de monitoreo de los impactos de las represas hidroeléctricas Jirau y Santo Antonio en territorio bolivinao: línea de base sobre ecosistemas y recursos acuáticos. Editorial INIA, Cochabamba, Bolivia.

Pereyra, G. A pesca na região de Madre Dios . Instituto de Investigações da Amazônia Peruana (Peru). In: Doria, C. R. C.; Athayde, S.; Dutka-Gianelli, J.; Luiz, A.M.T. Relatório: Seminário e Oficina Internacional Brasil, Bolívia e Peru: Desafios Nacionais e Internacionais de Gestão dos Recursos Pesqueiros na Bacia do Madeira. Ministério Público Estadual de Rondônia: Porto Velho, Maio de 2018.

PETESSE, M. L.; PETRERE, M. Tendency towards homogenization in fish assemblages 
in the cascade reservoir system of the Tietê river basin, Brazil. Ecological Engineering, v. 48, p. 109-116, 2012.

Pomeroy, R. S., \& Berkes, F. Two to tango: the role of government in fisheries comanagement. Marine Policy, v.21, n.5, p.465-480, 1997.

Raymond, C. M., Fazey, I., Reed, M. S., Stringer, L. C., Robinson, G. M., \& Evely, A. C. Integrating local and scientific knowledge for environmental management. Journal of environmental management, v.91, n.8, p. 1766-1777, 2010.

Reed, M. S. Stakeholder participation for environmental management: a literature review. Biological conservation, v.141, n,10, p. 2417-2431, 2008.

RAMOS, D., AMARAL, E., DORIA, C. R. C., LIMA, M. A. L., ROSSETE, A., ATHAYDE, S., MARQUES, E; ARAUJO, N.; BRASIL, W. O arcabouço dos Sistemas Socioecológicos como ferramenta para a análise dos impactos das barragens hidrelétricas no sistema da pesca artesanal na Amazônia. Revista Brasileira de Ciências Ambientais, In press.

Rodrigues, D. A. Presidente da Associação de pescadores do Rio Beni (Bolívia). In: Doria, C. R. C.; Athayde, S.; Dutka-Gianelli, J.; Luiz, A.M.T. Relatório: Seminário e Oficina Internacional Brasil, Bolívia e Peru: Desafios Nacionais e Internacionais de Gestão dos Recursos Pesqueiros na Bacia do Madeira. Ministério Público Estadual de Rondônia: Porto Velho, Maio de 2018.

SILVA, M. M. G. Reflexão dos pescadores da Vila Nova Teotônio. In: Doria, C. R. C.; Athayde, S.; Dutka-Gianelli, J.; Luiz, A.M.T. Relatório: Seminário e Oficina Internacional Brasil, Bolívia e Peru: Desafios Nacionais e Internacionais de Gestão dos Recursos Pesqueiros na Bacia do Madeira. Ministério Público Estadual de Rondônia: Porto Velho, Maio de 2018.

Soares-Filho, B.S., Nepstad, D.C., Curran, L.M., et al. Modelling conservation in the Amazon basin. Nature, n.440,p. 520-523, 2006.

SOUZA, I. Gestão de recursos pesqueiros na Reserva de Desenvolvimento Sustentável Mamirauá. In: Doria, C. R. C.; Athayde, S.; Dutka-Gianelli, J.; Luiz, A.M.T. Relatório: Seminário e Oficina Internacional Brasil, Bolívia e Peru: Desafios Nacionais e Internacionais de Gestão dos Recursos Pesqueiros na Bacia do Madeira. Ministério Público Estadual de Rondônia: Porto Velho, Maio de 2018.

TIMPE, K., KAPLAN D. 2017. The changing hydrology of a dammed Amazon. Science: Advances 3: 1-13. https://doi.org/10.1126/sciadv.1700611

Tedesco PA, Beauchard O, Bigorne R, Blanchet S, Buisson L, Conti L, Cornu J-F, Dias MS, Grenouillet G, Hugueny B, Jézéquel C, Leprieur F, Brosse S \& Oberdorff T. 2017. A global database on freshwater fish species occurrence in drainage basins. Scientific Data, n.4:17014.

Torrente-Vilara, G. Os peixes, as cachoeiras e as hidrelétricas. In: Doria, C. R. C.; Athayde, S.; Dutka-Gianelli, J.; Luiz, A.M.T. Relatório: Seminário e Oficina Internacional Brasil, Bolívia e Peru: Desafios Nacionais e Internacionais de Gestão dos Recursos Pesqueiros na Bacia do Madeira. Ministério Público Estadual de Rondônia: Porto Velho, Maio de 2018. 
Van Damme, P. Influencia de las represas hidroelectricas del rio Madera sobre el dorado y la pesca en la Amazonia boliviana. In: Doria, C. R. C.; Athayde, S.; Dutka-Gianelli, J.; Luiz, A.M.T. Relatório: Seminário e Oficina Internacional Brasil, Bolívia e Peru: Desafios Nacionais e Internacionais de Gestão dos Recursos Pesqueiros na Bacia do Madeira. Ministério Público Estadual de Rondônia: Porto Velho, Maio de 2018.

VELOSO, M. Presidente da Colônia de Pescadores de Porto Velho (Brasil) In: Doria, C. R. C.; Athayde, S.; Dutka-Gianelli, J.; Luiz, A.M.T. Relatório: Seminário e Oficina Internacional Brasil, Bolívia e Peru: Desafios Nacionais e Internacionais de Gestão dos Recursos Pesqueiros na Bacia do Madeira. Ministério Público Estadual de Rondônia: Porto Velho, Maio de 2018.

Winemiller, K.O. et al. (2016) Balancing hydropower and biodiversity in the Amazon, Congo, and Mekong. Science, n.351, p.128-129.

Yackulic, C. Programa de Gestão Adaptiva do Hidrelétrica do Glen Canyon (USGS - USA). In: Doria, C. R. C.; Athayde, S.; Dutka-Gianelli, J.; Luiz, A.M.T. Relatório: Seminário e Oficina Internacional Brasil, Bolívia e Peru: Desafios Nacionais e Internacionais de Gestão dos Recursos Pesqueiros na Bacia do Madeira. Ministério Público Estadual de Rondônia: Porto Velho, Maio de 2018. 